\title{
A CASE REPORT AND LITERATURE REVIEW ON PREGNANCY WITH ACUTE PROMYELOCYTIC LEUKEMIA WITH DIC
}

\author{
ARA T ${ }^{1}, \mathrm{KHAN} \mathrm{MA}^{2}$, AFROSE $\mathrm{S}^{3}$, NAZNEEN H${ }^{4}$, ALI $\mathrm{M}^{5}$, ISLAM MM ${ }^{6},{\text { AKTHER } \mathrm{M}^{7}, \text { AY MD. NAZIM }}^{8}$
}

\begin{abstract}
:
Acute promyelocytic leukemia (APL)-FAB-AML-M3 is now the most curable subtype of acute myeloblastic leukemia. The incidence of acute leukemia in pregnancy is rare. The occurrence of acute promyelocytic leukemia in pregnancy is also a very rare but management is very critical as APL is associated with life threatening hemorrhagic diathesis. Acute promyelocytic leukemia in pregnancy has not been reported in Bangladesh. Here we report a case of APL with DIC diagnosed during pregnancy. Clinical data and therapeutic approaches are presented. Her response to treatment was excellent resulted in a live birth and complete remission of her leukemia.
\end{abstract}

Key words: Acute promyelocytic leukemia, pregnancy, All trans-retinoic acid, Arsenic trioxide.

J Dhaka Med Coll. 2014; 23(2) : 262-265.

\section{Introduction:}

Acute promyelocytic leukemia (APL) is a subtype of AML in which a maturational arrest at promyelocyte series results in failure of normal hematopoiesis. It is an unique AML with distinct morphologic, cytogenetic and biologic characteristics. ${ }^{1}$ It is caused by reciprocal translocation between the long arms of chromosomes 15 and $17 .^{2}$ Clinically it is characterized by onset at a young age and a life threatening hemorrhagic complication that lead to DIC and hyperfibrinolysis.

The discovery of the molecular pathogenesis for APL has led to the targeted therapy; all-trans retinoic acid (ATRA) in the year 1988 with very high cure rate. ${ }^{3}$ In the early to mid 1990 s arsenic trioxide (ATO) has been added to the treatment of APL, first in relapsed patients then upfront. ${ }^{4}$

The incidence of leukemia in pregnancy is low. One in 75,000 to 100000 pregnancies $^{5}$. The occurrence of APL during pregnancy is also a rare event but management is very critical as APL is associated with life threatening hemorrhagic diathesis. Here we describe a patient with APL diagnosed during pregnancy that developed DIC, premature delivery and post partum hemorrhage. Management of this patient was discussed and a pertinent literature review of pregnancy in APL was discussed.

\section{Case Report:}

The patient was a 19years old house wife from lower socio-ecomonomic background, para 1, gravida 1 visted a local clinic at 33 weeks of gestation with a 1 month history of irregular fever not associated with chills and rigor relieved by taking antipyeretic, fatigue, episodic bleeding from various sites including purpuric rash, haematuria and rectal bleeding. She was in regular ante natal check up and took iron \& vitamin supplements.

1. Dr. Tasneem Ara, Associate Professor, Department of Hematology, Dhaka Medical College \& Hospital, Dhaka.

2. Dr. Mohiuddin Ahmed Khan, Professor of Hematology, Department of Hematology, Dhaka Medical College \& Hospital, Dhaka.

3. Dr. Salma Afrose, Professor of Hematology, Department of Hematology, Dhaka Medical College \& Hospital, Dhaka.

4. Dr. Md. Manirul Islam, Assistant Professor, Department of Hematology, Dhaka Medical College \& Hospital, Dhaka.

5. Dr. Mohammad Ali Medical Officer Department of Hematology, Dhaka Medical College \& Hospital, Dhaka.

6. Dr. Humayra Nazneen, Assistant Professor, Department of Hematology, Dhaka Medical College \& Hospital, Dhaka.

7. Dr. Maftuha Akhter, Assistant Professor, Department of Hematology, Dhaka Medical College \& Hospital, Dhaka.

8. Dr. Abu Yousuf Md. Nazim Uddin, Fellow Trainee, Department of Hematology, Dhaka Medical College \& Hospital, Dhaka.

Correspondence : Dr. Tasneem Ara, Associate Professor, Department of Hematology, Dhaka Medical College \& Hospital, Dhaka. 


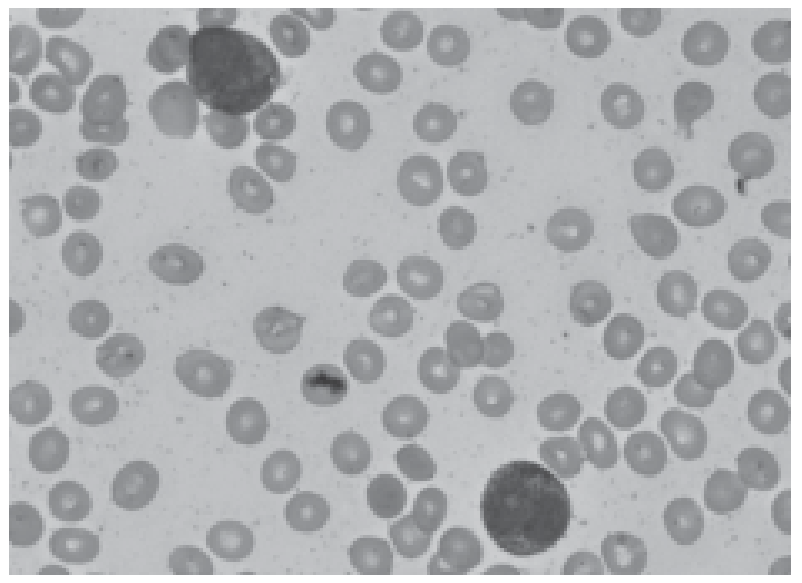

Fig.- 1: $P B F$ shows promyelocyte

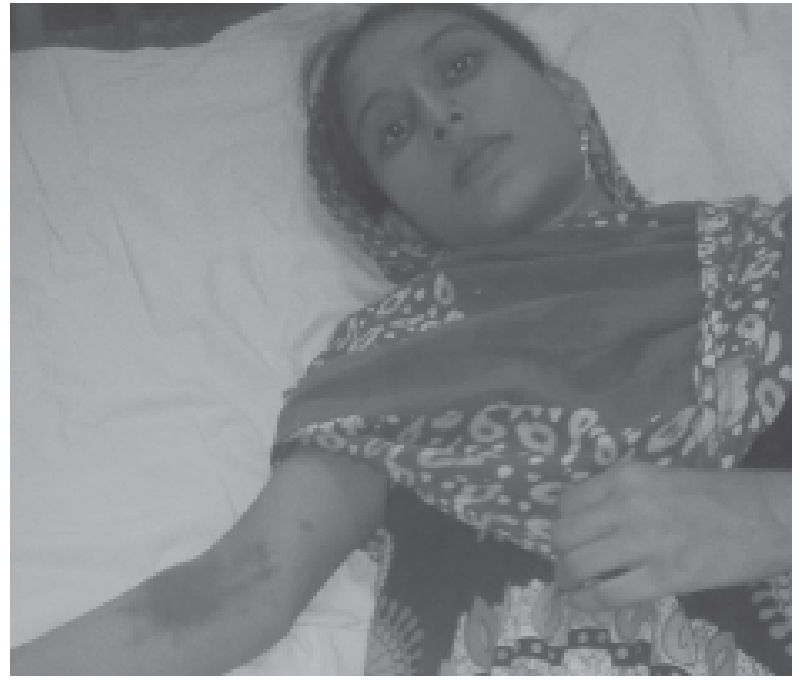

Fig.-2: Extensive ecyhmosis in patient of APL

With these complaints the patient was admitted at a local clinic and as part of emergency management she received several units of whole blood transfusion \& was referred to a general hospital. She was diagnosed there as acute leukemia on the basis of peripheral blood and supportive therapy was given. She received 6 units of fresh whole blood and 60 packs of (12 units) random donor platelet concentrate. As her condition deteriorated she was referred to the Department. of Hematology of Dhaka Medical College \& Hospital (tertiary $\&$ referral level hospital), on 05/09/10 for proper management; after 1 month she first developed bleeding manifestation.

Examination revealed multiple purpuric spots over both legs which were non palpable, leg edema, a temperature of $39^{\circ} \mathrm{C}$, pulse rate 92 beats/min, blood pressure 120/80 mm Hg. Height of the uterus was of 34 weeks size with cephalic presentation, Foetal heart rate was 144 beats/min \& regular. All other systemic examination revealed no abnormality. On investigation Hemoglobin $110.8 \mathrm{~g} / \mathrm{L}$ (after recieving several units of fresh whole blood from the referring hospital),leucocytes count $0.65 \times 10$ 9/L with a differential count of $30 \%$ segmented neutrophils,07\% lyphocytes, 03\% monocytes $60 \%$ promyelocytes and platelet count of $25 \times 10^{9} /$ L.Peripheral blood smear examination showed pancytopnia and left shift maturation of white blood cells with significant number of abnormal promyelocytes having characteristic immature bilobed nucleus and heavily granulated cytoplasm (Fig1), dagnostic of APL. Platelets were markedly reduced. Bone marrow examination showed replacement by abnormal hyper granular promyelocytes consistent with acute promyelocytic leukemia (APL). She was urgently started with ATRA $45 \mathrm{mg} / \mathrm{m}^{2} \mathrm{PO}$ in two divided doses daily. Molecular analysis by PCR revealed detection of PML-RARá short form. Screening test for DIC showed a prothrombin time of 16.7 seconds (control 11.8 seconds), activated partial thromboplastin time of 44.6 seconds (control 28 seconds),FDP10 $\mu \mathrm{gm} / \mathrm{dl}$ (Ref. value $<5 \mu \mathrm{gm} / \mathrm{dl}$ ), D-dimer: 1.0 $\mu \mathrm{gm} / \mathrm{dl}$ (Ref. value $<0.5 \mu \mathrm{gm} / \mathrm{dl}$ ). Biochemical analysis showed normal serum electrolytes, renal and liver function tests. USG of pregnancy profile 34 weeks of single live foetus and liquor was adequate. The patient was diagnosed as a case of intermediate risk Acute promyelocytic leukaemia with 34 wks pregnancy with DIC a diagnosis not suspected by the referring clinic. For neutropenic fever patient received broad spectrum injectable antibiotics (Ceftazidime and metronidazole) empirically, and then injection Meropenem was given as per the blood $\mathrm{C} / \mathrm{S}$ report resulting in resolution of fever. Patient also received 1 unit of red cell concentrate, 6 pack platelet rich plasma (PRP), 4units of fresh frozen plasma. On 3rd day of ATRA the patient developed labour pain, and a baby girl was delivered by normal vaginal delivery, placenta was removed by controlled cord traction. The birth weight was 
1600gm and no physical abnormalities were detected. After delivery, per vaginal bleeding was started in copious amount which was controlled by intra uterine condom catheterisation with ballon inflation. 3 units of red cell concentrate and one unit of random donor platelet concentrate were transfused. Specific treatment of APL (ATRA) was continued. As per our institutional protocol after delivery she was started with 2 hours intravenous infusion of $0.15 \mathrm{mg} / \mathrm{m} 2$ dose of arsenic trioxide .ATRA was discontinued after 10 days. Breast feeding was withheld and milk suppression was done by tablet bromocriptine. Result of induction with ATRA followed by ATO was complete remission (CR) with full haematological recovery. Repeat bone marrow at day 45 confirmed complete haematological and cytogenetic remission. The patient then received single course of standard consolidation by ATO (two hour intravenous infusion five days a week schedule for five weeks).After consolidation therapy patient was evaluated for molecular remission and was found PCR negative for PML-RARá fusion gene transcript. Then standard maintenance therapy by ATRA+oral chemotherapy (ATRA $45 \mathrm{mg} / \mathrm{m}^{2} /$ day for 15 day every 3 months plus 6-MP 60mg / $\mathrm{M}^{2} /$ day PO plus methotrexate $20 \mathrm{mg} / \mathrm{m}^{2} \mathrm{PO}$ weekly) was started and continued for 2 years. At the time of writing, the patient has completed her maintenance chemotherapy. She and her 3 and a half years old female child are well. One year back she gave birth to a male healthy boy.

\section{Discussion:}

The occurrence of acute leukemia in pregnancy is very rare, with an estimated incidence of one in 100000 pregnancies annually. ${ }^{5}$ APL in pregnancy is a rare event, with fewer than 60 case reports. Early diagnosis $\&$ immediate installation of specific treatment is most important. Management of leukemia during pregnancy represents a great challenge, because chemotherapeutic agents have potential teratogenic effects. Prior to the introduction of ATRA, induction with standard chemotherapy in APL resulted in few life birth. ${ }^{6}$ The successful outcome of APL in pregnancy using ATRA was first reported in the year
1994. ${ }^{7}$ After then use of ATRA alone or in combination with standard chemotherapy resulted in a favourable outcome. Several studies demonstrated ATRA seemed to be reasonably safe during the second and third trimester of pregnancy in the treatment of APL, although known to be exhibit severe teratogenic effects during the first trimester of pregnancy. $8,9,10,11$

In the present case the patient had delayed diagnosis. A longer time has been elapsed between admission to general hospital and arrival to the tertiary level hospital. But the patient survived as adequate supportive care with blood and blood product was given in the general hospital.

Management of APL in pregnancy is always a cause for major concern because APL is a hematologic emergency with high risk of mortality associated with DIC. Moreover pregnancy in patients with APL requires special consideration to increase the probability of survival of both mother and fetus. Our patient also presented with DIC.

In our institution we treat APL with single agent ATO alone. However in this case initial treatment was started with ATRA because safety of ATO in pregnancy has not been proved rather it has been shown to be highly embryo toxic in several animal models. ${ }^{12}$ Although human data are limited low birth weight, spontaneous abortion and still birth are reported among people exposed to arsenic from drinking water. ${ }^{13}$ While conventional chemotherapy has been shown to exacerbate DIC ATRA improve DIC. ${ }^{14}$ At day 3 of induction with ATRA the patient developed labour pain spontaneously. Data regarding induction of labour pain by ATRA is not adequate. One case report demonstrated spontaneous premature labor with ATRA. ${ }^{15}$ However Toronto Leukemia Study group showed an increased risk of premature delivery as well as small for date babies who received chemotherapy during their pregnancies. ${ }^{16}$ As per our institutional protocol she was given standard induction by Arsenic trioxide(ATO).There are several published paper demonstrated successful induction and consolidation by ATO alone in newly diagnosed cases of APL. ${ }^{17,18,19}$ 
After delivery, the patient developed severe post partum hemorrhage which was managed jointly by the hematologist and the obstetrician. Her bleeding was reduced after three days.

\section{Conclusion:}

Acute leukemia is an extremely aggressive disease and is fatal unless promptly treated. Pregnancy does not alter the course of acute leukemia and APL is among the most treatable disease of hematologic malignancy. Fetal concerns should not delay therapy this may proved to be life saving particularly if the leukemia happens to be APL a cancer with very high cure rate. Multidisciplinary approach involving hematologist and obstetrician in the management of APL in pregnancy can ensure a good outcome for both the mother and the fetus despite the life threatening consequences of DIC which is common at diagnosis in most cases of APL. Our patient's case also showed fertility preservation following treatment with arsenic trioxide. She became pregnant two years after completion of the maintenance chemotherapy of APL.

\section{References:}

1. Stone RM,Mayer RJ. The unique aspects of acute promyelocytic leukemia. J Clin Oncol. 1990; 8(11):1913-1921.

2. Larson RA,Kondo K,Vardiman JW, ButlerAE, Golomb HM, Rowly JD.Evidence for a 15;17 translocation in every patient with acute promyelocytic leukemia.Am J Med.1984;76(5):827841.

3. Huang ME, Ye YC,Chen SR,et al.Use of all-trans retinoic acid in the treatment of acute promyelocytic leukemia..Blood, 1988; 72:567572 .

4. ShenZX, Shi ZZ,Fang J,et al.All trans retinoic acid /arsenic trioxide combination yields a high quality remission and survival in newly diagnosed acute promyelocytic leukemia.Proc Natl Acad Sci USA.2004;101:5328-5335.

5. Litchman M,Liesveld J:Acute myelogenous leukemia.In Williams Hematology. $6^{\text {th }}$ edition.New York,NY:Mc-Graw Hill;2001:1047-1084.

6. Catanzarite VA, Ferguson JE. Acute leukemia and pregnancy: a review of management and outcome, 1972-82. Obstet Gynecol Surv 1984;39:663-678.
7. Giagounidis AAN, Beckmann MW, Giagounidis AS, Aivado M, Emde T, Germing U, Riehs T, Heyll A, Aul C. Acute promyelocytic leukemia and pregnancy. Eur J Haematol 2000; 64: 267-271.

8. Stenloft J,Hvidman LE,All-transretinoic acid in acute promyelocytic leukemia in late pregnancy [abstract].Leukemia1994;8:1585-1588.

9. Incerpi MH, Miller DA, Posen R, Byrne JD. Alltrans retinoic acid for the treatment of acute promyelocytic leukemia in pregnancy. Obstet Gynecol 1997; 89: 826-828. Harrison P, Chipping P, Fothergill GA.

10. Successful use of all-transretinoic acid in acute promyelocytic leukaemia during the second trimester of pregnancy. Br J Haematol 1994; 86: 681-682.

11. Celo JS, Kim HC, Houlin C, Canavan BF, Manzullo GP, Saidi P.Acute promyelocytic leukemia in pregnancy: all-trans retinoic acid as a newer therapeutic option. Obstet Gynecol 1994; 83: 808811.

12. Peterkova R and L.Puzanova.Effect of trivalent and pentavalent arsenic on early developmental stages of the chick embryo. Folia morphologica 1974;24;743-750.

13. U.S Environmental Protection agency.Arsenic compounds. http://www.epa.gov. Accessed January 8, 2008.

14. Frankel SR,, Eardly A,Lauwers J,Weiss M, Warrel RP.The retinoic acid syndrome in acute promyelocytic leukemia.Ann Inter Med 1992; 117:292-296.

15. Sham RL. All-trans retinoic acid-induced labor in a pregnant patient with acute promyelocytic leukemia. Am J Hematol 1996; 53: 145.

16. Reynos,FA Shepherd,HA Messner et al, the Toronto Leukemia Study Group experience with long term follow-up of children exposed in utero to chemotherapeutic agents for acute leukemia during pregnancy.J Clin Oncol 1987;51:10981106.

17. Sun HD, L Hu XC,Zhang TD,Ai -Lin I treated 32 cases of acute promyelocytic cases of leukemia.Chin J Integral Chin West Med.1992; 12:170.

18. George B,Mathews V,Vishwabandhya A,Srivastava A, Chandy M.Arsenic trioxide in the treatment of patients with newly diagnosed acute promyelocytic leukemia:toxicity and outcome.Blood. 2004; 104: 254a.

19. Gavamzadeh A,Alimoghaddam K, Ghaffari H, et al.Treatment of new cases of acute promyelocytic leukemia by arsenic trioxide without ATRA and/ or chemotherapy.Annals of Oncology 17:131134,2006 . 